\title{
Nonlinear Mathematical Model of Interference of Fundamental and Applied Researches
}

\author{
Chilachava Temur ${ }^{1}$, Gvinjilia Tsira ${ }^{2}$ \\ ${ }^{1}$ Departament of Applied Mathematics, Sokhumi State University, Tbilisi, Georgia \\ ${ }^{2}$ Department of Exact and Natural Sciences, Batumi State Maritime Academy, Batumi, Georgia
}

Email address:

temo_chilachava@yahoo.com (C. Temur), Gvinjilia1959@mail.ru (G. Tsira)

\section{To cite this article:}

Chilachava Temur, Gvinjilia Tsira. Nonlinear Mathematical Model of Interference of Fundamental and Applied Researches. International Journal of Systems Science and Applied Mathematics. Vol. 2, No. 6, 2017, pp. 110-115. doi: 10.11648/j.ijssam.20170206.11

Received: August 23, 2017; Accepted: September 25, 2017; Published: November 2, 2017

\begin{abstract}
In work the new nonlinear continuous mathematical model of interference of fundamental and applied researches on the example of one, perhaps closed for external customers, of scientifically - research institute (micro-model) is considered. For a special case, Cauchy's problem for nonlinear system of differential equations of first order is definitely decided analytically. In more general case based on Bendikson's criteria the theorem of not existence in the first quarter of the phase plane of solutions of closed integral curves is proved. Conditions on model parameters in case of which existence of limited solutions of system of nonlinear differential equations is possible are found.
\end{abstract}

Keywords: Nonlinear Mathematical Model, Fundamental and Applied Researches, Phase Plane, Bendikson's Criteria

\section{Introduction}

Mathematical and computer modeling has been widely recognized in such disciplines as sociology, history, political science, and others $[1,2]$. There is an interest in creation of a mathematical model, which would give the opportunity to determine the dynamics of changes in the number of voters of political subjects during the election period. Elections can be divided into two parts: the two-party and multi-party elections.

In [3 - 5] quantities of information streams by means of new mathematical models of information warfare are studied. By information warfare the authors mean an antagonism by means of mass media (an electronic and printing press, the Internet) between the two states or the two associations of states, or the economic structures (consortiums) conducting purposeful misinformation, propagation against each other.

It was shown that in case of high aggression of the contradictory countries, not preventive image the operating peacekeeping organizations won't be able to extinguish the expanding information warfare.

In works $[6,7]$ linear and nonlinear mathematical models of information warfare, and also optimizing problems are considered.
In [8] the new nonlinear mathematical and computer model of information warfare with participation of interstate authoritative institutes is offered. The model is described by Cauchy's problem for nonlinear non-homogeneous system of the differential equations. Confronting sides in extend of provocative statements, the third side (the peacekeeping international organizations) extends of soothing statements, interstate authoritative institutes the peacekeeping statements call the sides for the termination of information warfare. In that specific case, modes of information warfare "aggressorvictim", for the third peacekeeping side are received exact analytical solutions, and functions defining number of the provocative statements distributed by the antagonistic sides satisfy to Cauchy's problems for Riccati certain equations which are solved by a numerical method. For the general model computer modeling is carried out and shown that irrespective of high aggression of confronting sides, interstate authoritative institutes will be able to extinguish information warfare and when for this purpose efforts of only the international organizations insufficiently.

The article $[9,10]$ concerning of Chilker task is entered refers to the boundary value problem for a system of ordinary differential equations and optimal control problem. In Chilker tasks right boundary conditions are set in different, uncommitted time points for different coordinates of the 
unknown vector - functions. Proposed methods solutions of Chilker tasks.

These papers [11 - 13] present the nonlinear mathematical model of the public or the administrative management (or the macro and micro model). The cases of both constant and variable pressure forces on freethinking people were analyzed. Exact analytical decisions which determine dynamics of a spirit both free-thinking people, and operated (conformists) of people by time are received. During these analyses various governance systems were considered: a liberal, democratic, semi dictatorial and dictatorial.

These works [14 - 18] considered a two or three-party (one pro-government and two opposition parties) nonlinear mathematical model of elections when coefficients are constant. The assumption was made that the number of voters remains the same between two consecutive elections (zero demographic factor of voters). The exact analytical solutions were received. The conditions under which opposition party can win the upcoming elections were established.

These works [19 - 21] considered a two-party (progovernment and opposition parties) nonlinear mathematical model of elections with variable coefficients.

In work [22] proposed the nonlinear mathematical model with variable coefficients in the case of three-party elections, that describes the dynamics of the quantitative change of the votes of the pro-government and two opposition parties from election to election. The model takes into account the change in the total number of voters in the period from election to election, i.e. the so-called demographic factor during the elections is taken into account. The model considered the cases with variable coefficients. In the particular case obtained exact analytical solutions. The conditions have been identified under which the opposition can win the forthcoming elections, and in some cases, the progovernment party can stay in power. In general Cauchy problem was solved numerically using the MATLAB software package.

In [23] consider the nonlinear mathematical model of bilateral assimilation without demographic factor. It was shown that the most part of the population talking in the third language is assimilated by that widespread language which speaks bigger number of people (linear assimilation). Also it was shown that in case of zero demographic factor of all three subjects, the population with less widespread language completely assimilates the states with two various widespread languages, and the result of assimilation (redistribution of the assimilated population) is connected with initial quantities, technological and economic capabilities of the assimilating states.

In [24] mathematical modeling of nonlinear process of assimilation taking into account demographic factor is offered. In considered model taking into account demographic factor natural decrease in the population of the assimilating states and a natural increase of the population which has undergone bilateral assimilation is supposed. At some ratios between coefficients of natural change of the population of the assimilating states, and also assimilation coefficients, for nonlinear system of three differential equations are received the two first integral. Cases of two powerful states assimilating the population of small state formation (autonomy), with different number of the population, both with identical and with various economic and technological capabilities are considered. It is shown that in the first case the problem is actually reduced to nonlinear system of two differential equations describing the classical model "predator - the victim". Thus, naturally a role of the victim plays the population which has undergone assimilation, and a predator role the population of one of the assimilating states. The population of the second assimilating state in the first case changes in proportion (the coefficient of proportionality is equal to the relation of the population of assimilators in an initial time point) to the population of the first assimilating side. In the second case the problem is actually reduced to nonlinear system of two differential equations describing type model "a predator - the victim", with the closed integrated curves on the phase plane. In both cases there is no full assimilation of the population to less widespread language. Intervals of change of number of the population of all three objects of model are found. The considered mathematical models which in some approach can model real situations, with the real assimilating countries and the state formations (an autonomy or formation with the unrecognized status), undergone to bilateral assimilation, show that for them the only possibility to avoid from assimilation is the natural demographic increase in population and hope for natural decrease in the population of the assimilating states.

In [25] mathematical modeling of nonlinear process of the assimilation taking into account positive demographic factor which underwent bilateral assimilation of the side and zero demographic factor of the assimilating sides is considered. In model three objects are considered: the population and government institutions with widespread first language, influencing by means of state and administrative resources on the population of the third state formation for the purpose of their assimilation; the population and government institutions with widespread second language, influencing by means of state and administrative resources on the population of the third state formation for the purpose of their assimilation; population of the third state formation which is exposed to bilateral assimilation from two powerful states or the coalitions.

For nonlinear system of three differential equations of the first order are received the two first integral. Special cases of two powerful states assimilating the population of small state formation (autonomy), with different initial number of the population, both with identical and with various economic and technological capabilities are considered. It is shown that in all cases there is a full assimilation of the population to less widespread language. Thus, proportions in which assimilate the powerful states the population of small state formation are found.

In [26] the model it is supposed that the powerful state with a widespread state language carries out assimilation of 
the population of less powerful state and the third population talking in two languages, different in prevalence. Carries out assimilation of the population of the state formation with the least widespread language to the turn, less powerful state. Not triviality of model assumes negative demographic factor of the powerful state-assimilating and positive demographic factor of the state formation which is under bilateral assimilation. For some ratios between demographic factors of the sides and coefficients of assimilations, for nonlinear system of three differential equations with the corresponding conditions of Cauchy the first integrals are found. In particular, in the first case the first integral in space of required functions represents a hyperbolic paraboloid, and in the second case - a cone. In these cases, the nonlinear system of three differential equations is reduced to nonlinear system of two differential equations for which the second first integrals are found and in the phase plane of decisions are investigated behavior of integrated curves. In more general case with application of a criteria of Bendikson the possibility of existence of the closed integrated curves is proved that indicates a possibility of a survival of the population finding under double assimilation.

One of the perspective and quickly field of application of mathematical modeling is dynamics of innovative processes. Researches in this area show that the crisis phenomena have not the casual, but systematic character defined by the determined mechanisms. Therefore many features of behavior of innovative processes can be described within the determined systems of the differential equations. The difficult behavior of these systems, including selforganization processes, gives in to the description thanks to existence of the nonlinear members who are present at mathematical models of dynamic systems. Research of mathematical models of innovative processes in scientific and educational areas is of a great interest [2].

In [27] work the nonlinear mathematical model of dynamics of processes of cooperation interaction in innovative system: fundamental researches - applied researches - developmental works - innovations is offered.

In [28] the new nonlinear mathematical model of interaction of fundamental and applied researches is considered.

\section{Method}

Unlike mathematical modeling of physical processes where at creation of model it is necessary to consider implementation of five classical laws, at creation of mathematical model of social processes of such general method doesn't exist. Therefore at creation of the mathematical model describing this or that social process it is necessary to consider some analogies and a certain adequacy of the system of the equations describing this process.

The new nonlinear continuous mathematical model of interference of the fundamental and applied researches conducted in one scientifically - research institute has an appearance (micro-model):

$$
\left\{\begin{array}{c}
\frac{d u(t)}{d t}=\alpha_{1}(t) u-\beta_{1}(t) u^{2}+\gamma_{12}(t) u v+\delta_{1}(t)-\delta_{2}(t) \\
\frac{d v(t)}{d t}=\alpha_{2}(t) v-\beta_{2}(t) v^{2}+\gamma_{21}(t) u v \\
u(0)=u_{0}, v(0)=v_{0}
\end{array}\right.
$$

$u(t), v(t)$-according to the number of fundamental and applied researches in time-point $t ; \alpha_{1}(t), \alpha_{2}(t)$-respectively growth rates of number of fundamental and applied researches in time-point $t ; \beta_{1}(t), \beta_{2}(t)$-respectively coefficients of super-saturation of number of fundamental and applied researches in time-point $t$;

$\gamma_{12}(t), \gamma_{21}(t)$-respectively coefficients of interference of fundamental and applied researches in time-point $t$; $\delta_{1}(t), \delta_{2}(t)$ - according to the speed of inflow and outflow of fundamental researches to time-point $t$;

$$
\alpha_{1}(t), \alpha_{2}(t), \quad \beta_{1}(t), \beta_{2}(t), \quad \gamma_{12}(t), \gamma_{21}(t), \quad \delta_{1}(t), \delta_{2}(t) \quad-
$$
positive functions on a segment $t \in[0, T]$, $u(t), v(t) \in C^{1}[0, T]$.

Let's consider a special case

$$
\begin{gathered}
\delta_{1}(t)=\delta_{2}(t), \\
\alpha_{1}(t)=\alpha_{1}=\text { cons } t>0, \alpha_{2}(t)=\alpha_{2}=\text { cons } t>0 \\
\beta_{1}(t)=\beta_{1}=\text { const }>0, \beta_{2}(t)=\beta_{2}=\text { const }>0, \\
\gamma_{12}(t)=\gamma_{12}=\text { cons } t>0, \gamma_{21}(t)=\gamma_{21}=\text { const }>0 .
\end{gathered}
$$

Then from (1)- (3) we will receive the following Cauchy's problem for nonlinear system of the differential equations

$$
\left\{\begin{array}{l}
\frac{d u(t)}{d t}=\alpha_{1} u(t)-\beta_{1} u^{2}(t)+\gamma_{12} u(t) v(t) \\
\frac{d v(t)}{d t}=\alpha_{2} v(t)-\beta_{2} v^{2}(t)+\gamma_{21} u(t) v(t)
\end{array}\right.
$$

$$
u(0)=u_{0}, v(0)=v_{0}
$$

Let's find the stationary nontrivial solution of system (4) which corresponds to performance of the following system

$$
\left\{\begin{array}{l}
\frac{d u(t)}{d t}=0 \\
\frac{d v(t)}{d t}=0
\end{array}\right.
$$

Then from (4) we will receive non-homogeneous system of the linear algebraic equations for $(u, v)$ functions 


$$
\left\{\begin{array}{l}
-\beta_{1} u+\gamma_{12} v=-\alpha_{1} \\
\gamma_{21} u-\beta_{2} v=-\alpha_{2}
\end{array}\right.
$$

According to Kramer's formulas we will calculate the corresponding determinants

$$
\begin{aligned}
\Delta & =\left|\begin{array}{cc}
-\beta_{1} & \gamma_{12} \\
\gamma_{21} & -\beta_{2}
\end{array}\right|=\beta_{1} \beta_{2}-\gamma_{12} \gamma_{21} \\
\Delta_{1} & =\left|\begin{array}{cc}
-\alpha_{1} & \gamma_{12} \\
-\alpha_{2}-\beta_{2}
\end{array}\right|=\alpha_{1} \beta_{2}+\alpha_{2} \gamma_{12}>0 \\
\Delta_{2} & =\left|\begin{array}{l}
-\beta_{1}-\alpha_{1} \\
\gamma_{21}-\alpha_{2}
\end{array}\right|=\alpha_{2} \beta_{1}+\alpha_{1} \gamma_{21}>0
\end{aligned}
$$

Then the solution of system (6) owing to (7) exists only when it is executed

$$
\Delta=\left|\begin{array}{c}
-\beta_{1} \gamma_{12} \\
\gamma_{21}-\beta_{2}
\end{array}\right|=\beta_{1} \beta_{2}-\gamma_{12} \gamma_{21} \neq 0
$$

and the stationary nontrivial decision of system (4) will register in the following look

$$
u_{s t}=\frac{\Delta_{1}}{\Delta}, v_{s t}=\frac{\Delta_{2}}{\Delta}
$$

According to (7), (8) in order that this stationary solution was found in the first quarter of the phase plane $(O, u, v)$, performance of the following inequality is necessary

$$
\Delta=\beta_{1} \beta_{2}-\gamma_{12} \gamma_{21}>0
$$

Thus, when performing inequality on constant models (9), the system of the differential equations (4) has the nontrivial stationary decision in the first quarter (other quarters don't make physical sense) the phase plane $(O, u, v)$

$$
u=u_{s t}=\frac{\alpha_{1} \beta_{2}+\alpha_{2} \gamma_{12}}{\beta_{1} \beta_{2}-\gamma_{12} \gamma_{21}}, v=v_{s t}=\frac{\alpha_{2} \beta_{1}+\alpha_{1} \gamma_{21}}{\beta_{1} \beta_{2}-\gamma_{12} \gamma_{21}}
$$

For a special case when conditions are satisfied ((9) in this case it isn't satisfied)

$$
\alpha_{1}=\alpha_{2}, \beta_{1}=\gamma_{21}, \beta_{2}=\gamma_{12}
$$

Cauchy's task (4), (5) is definitely decided analytically. Really, (4), (5), taking into account (11) will take a form

$$
\left\{\begin{array}{l}
\frac{d u(t)}{d t}=\alpha_{1} u(t)-\beta_{1} u^{2}(t)+\beta_{2} u(t) v(t) \\
\frac{d v(t)}{d t}=\alpha_{1} v(t)-\beta_{2} v^{2}(t)+\beta_{1} u(t) v(t)
\end{array}\right.
$$

$$
u(0)=u_{0}, v(0)=v_{0}
$$

Multiplying the first equation of system (12) on $v(t)$, and the second on $u(t)$, and then added the received expressions, we will receive the first integral of system (12)

$$
u(t) v(t)=u_{0} v_{0} e^{2 \alpha_{1} t}
$$

Substituting the first integral (13) in the first equation of system (12), for unknown function $u(t)$ we will receive Ricatti's equation

$$
\frac{d u(t)}{d t}=\alpha_{1} u(t)-\beta_{1} u^{2}(t)+\beta_{2} u_{0} v_{0} e^{2 \alpha_{1} t}
$$

It is known that generally in quadratures Ricatti's equation isn't solved as knowledge of one particular decision is necessary.

It is easily possible to check that the following function $u_{1}(t)$ is the particular decision equations (14)

$$
u_{1}(t)=\sqrt{\frac{\beta_{2} u_{0} v_{0}}{\beta_{1}}} e^{\alpha_{1} t}
$$

Then entering the transformation canceling the nonhomogeneous member in the equation (14)

$$
u(t)=u_{1}(t)+z(t)
$$

from (14) - (16) for function $z(t)$ we will receive Bernoulli's equation

$$
\frac{d z(t)}{d t}=\left(\alpha_{1}-2 \beta_{1} u_{1}\right) z(t)-\beta_{1} z^{2}(t)
$$

Entering transformation

$$
y(t)=\frac{1}{z(t)}
$$

for $y(t)$ function we will receive the non-homogeneous linear equation of the first order

$$
\frac{d y(t)}{d t}=-\left(\alpha_{1}-2 \beta_{1} u_{1}\right) y(t)+\beta_{1}
$$

which common decision has an appearance

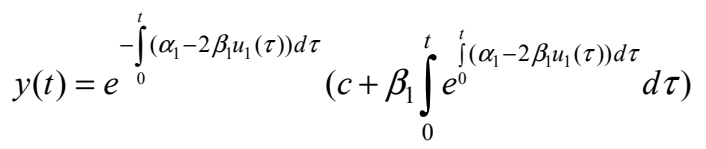

Considering (12), (15), (16), (18) for $y(t)$ function we will receive an initial condition

$$
y(0)=\frac{1}{z(0)}=\frac{1}{u_{0}-\sqrt{\frac{\beta_{2} u_{0} v_{0}}{\beta_{1}}}}, u_{0} \neq \frac{\beta_{2} v_{0}}{\beta_{1}}
$$

taking into account which we will receive the only decision 
for $y(t)$ function

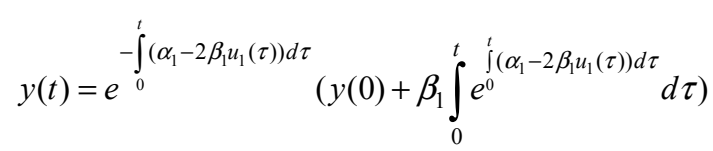

Respectively, it agrees (13), (16), (18) we will define required $u(t), v(t)$ functions Cauchy's tasks (12)

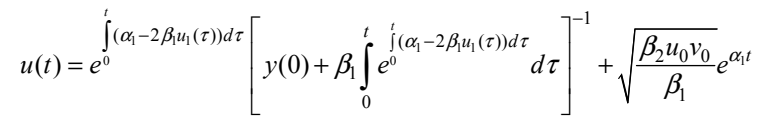

$$
\begin{aligned}
& v(t)=u_{0} v_{0} e^{2 \alpha_{1} t} u^{-1}(t)
\end{aligned}
$$

From (21) it is possible to find an asymptotics of $u(t), v(t)$ decisions at big times

$$
\begin{aligned}
& u(t) \approx \sqrt{\frac{\beta_{2} u_{0} v_{0}}{\beta_{1}}} e^{\alpha_{1} t}, t \rightarrow \infty \\
& v(t) \approx \sqrt{\frac{\beta_{1} u_{0} v_{0}}{\beta_{2}}} e^{\alpha_{1} t}, t \rightarrow \infty
\end{aligned}
$$

Let's rewrite system of the equations (4) in a vector form

$$
\begin{gathered}
\frac{d \vec{w}(t)}{d t}=\vec{F}, \\
\vec{w}(t)=\left(\begin{array}{c}
u(t) \\
v(t)
\end{array}\right), \vec{F}=\left(\begin{array}{c}
F_{1} \\
F_{2}
\end{array}\right),
\end{gathered}
$$

where designations are entered

$$
\begin{gathered}
F_{1}(u, v) \equiv \alpha_{1} u(t)-\beta_{1} u^{2}(t)+\gamma_{12} u(t) v(t) \\
F_{2}(u, v) \equiv \alpha_{2} v(t)-\beta_{2} v^{2}(t)+\gamma_{21} u(t) v(t)
\end{gathered}
$$

Theorem. When performing system of inequalities on constant models

$$
\left\{\begin{array}{l}
\gamma_{12} \geq 2 \beta_{2} \\
\gamma_{21} \geq 2 \beta_{1}
\end{array}\right.
$$

in the first quarter of the $(O, u, v)$ phase plane of solutions of a task of Cauchy (4) there is no closed integrated curve entirely lying in this area.

Proof. Let's consider divergence of the vector field $\vec{F}$

$$
\begin{gathered}
\operatorname{div} \vec{F}=\nabla^{i} F_{i}=\frac{\partial F_{1}}{\partial u}+\frac{\partial F_{2}}{\partial v}=\alpha_{1}-2 \beta_{1} u(t)+\gamma_{12} v(t)+\alpha_{2}-2 \beta_{2} v(t)+\gamma_{21} u(t) \\
\operatorname{div} \vec{F}=\alpha_{1}+\alpha_{2}+\left(\gamma_{21}-2 \beta_{1}\right) u(t)+\left(\gamma_{12}-2 \beta_{2}\right) v(t)
\end{gathered}
$$

It is clear, that owing to inequalities (24), and also a natural assumption $\alpha_{1}>0, \alpha_{2}>0$, in the first quarter of the $(O, u, v)$ phase plane of decisions

$$
\operatorname{div} \vec{F}=\alpha_{1}+\alpha_{2}+\left(\gamma_{21}-2 \beta_{1}\right) u(t)+\left(\gamma_{12}-2 \beta_{2}\right) v(t)>0
$$

i.e. divergence of the $\vec{F}$ vector field doesn't change a sign, and then owing to Bendikson's criteria there is no closed integrated curve entirely lying in this area.

The theorem is proved.

\section{Result}

In work the new nonlinear continuous mathematical model of interference of fundamental and applied researches on the example of one, perhaps closed for external customers, of scientifically - research institute (micro-model) is considered. For a special case, Cauchy's problem for nonlinear system of differential equations of first order is definitely decided analytically. In more general case based on Bendikson's criteria the theorem of not existence in the first quarter of the phase plane of solutions of closed integral curves is proved. Conditions on model parameters in case of which existence of limited solutions of system of nonlinear differential equations is possible are found.

\section{Discussion}

From our point of view, the new mathematical model of interference of basic and applied researches offered by us, allows to describe this process which takes place at the mathematical level and to show some quantitative communications between them.

\section{Conclusion}

The offered new nonlinear mathematical model allows to estimate influence of fundamental and applied researches (works) at each other, to find conditions on constant models in case of which there can be limited decisions, i.e. closed integral curves in the first quadrant of the phase plane of decisions.

\section{References}

[1] Samarski A. A., Mihailov A. P. Mathematical modeling. Moskow, Fizmatlit, 2006 (russian).

[2] Chilachava T. I., Dzidziguri Ts. D. Mathematical modeling. Tbilisi, Inovacia, 2008, (georgian).

[3] Chilachava T. I., Kereselidze N. G. Mathematical modeling of the information warfare. Georgian Electronic Scientific Journal: Computer Science and Telecommunications, 2010, № 1 (24), pp. 78-105 (georgian).

[4] Chilachava T. I., Kereselidze N. G. Non-preventive continuous linear mathematical model of information warfare. Sokhumi State University Proceedings, Mathematics and Computer Sciences, 2009, № VII, pp. 91-112.

[5] Chilachava T. I., Kereselidze N. G. Continuous linear mathematical model of preventive information warfare. Sokhumi State University Proceedings, Mathematics and Computer Sciences, 2009, № VII, pp. 113-141. 
[6] Chilachava T. I., Kereselidze N. G. Optimizing Problem of Mathematical Model of Preventive Information Warfare, Informational and Communication Technologies - Theory and Practice: Proceedings of the International Scientific Conference ICTMC- 2010 USA, Imprint: Nova, 2011, pp. $525-529$.

[7] ChilachavaT. I., Kereselidze N. G. Mathematical modeling of information warfare. Information warfare, 2011, №1(17), стр. 28-35 (russian).

[8] Chilachava T. I., Chakhvadze A. Continuous nonlinear mathematical and computer model of information warfare with participation of authoritative interstate institutes. Georgian Electronic Scientific Journal: Computer Science and Telecommunications, 2014, № 4(44), pp. 53-74.

[9] Kereselidze N. An optimal control problem in mathematical and computer models of the information warfare. Differential and Difference Equations with Applications: ICDDEA, Amadora, Portugal, May 2015, Selected Contributions. Springer Proceedings in Mathematics \& Statistics, 164, DOI 10.1007/978-3-319-32857-7_28, Springer International Publishing Switzerland 2016, pp. 303-311.

[10] Kereselidze N. G. Chilker's type mathematical and computer models in the information warfare. Information warfare, 2016, № 2 (38), pp. 28-35 (russian).

[11] Chilachava T. I., Dzidziguri Ts. D., Sulava L. O., Chakaberia M. R. Nonlinear mathematical model of administrative management. Sokhumi State University Proceedings, Mathematics and Computer Sciences, vol. VII, 2009, pp.169180 (georgian).

[12] Chilachava T. I., Sulava L. O., Chakaberia M. R. On some nonlinear mathematical model of administration. Problems of security management of complex systems. Proceedings of the XVIII International Conference, Moscow, 2010, pp. 492-496 (russian).

[13] Chilachava T. I., SulavaL. O. A nonlinear mathematical model of management. Georgian Electronic Scientific Journal: Computer Science and Telecommunications, 2013, №1(37) pp 60-64 (russian).

[14] Chilachava T. I. Nonlinear mathematical model of the dynamics of the voters pro-government and opposition parties (the two election subjects) Basic paradigms in science and technology. Development for the XXI century. Transactions II. 2012, pp. 184-188 (russian).

[15] Chilachava T. I Nonlinear mathematical model of the dynamics of the voters pro-government and opposition parties. Problems of security management of complex systems. Proceedings of the XX International Conference, Moscow, 2012, pp. 322-324 (russian).

[16] Chilachava T. I. Nonlinear mathematical model of dynamics of voters of two political subjects. Seminar of the Institute of Applied Mathematics named I. Vekua Reports, 2013, vol. 39, pp. 13-22.

[17] Chilachava T. I. Nonlinear mathematical model of three-party elections. Problems of security management of complex systems. Proceedings of the XXI International Conference, Moscow, 2013, pp. 513-516.

[18] Chilachava T. I., Chochua Sh. G. Nonlinear mathematical model of two-party elections in the presence of election fraud. Problems of security management of complex systems. Proceedings of the XXI International Conference, Moscow, 2013, pp. 349-352 (russian).

[19] Chilachava T. I., Sulava L. O. Mathematical and computer modeling of nonlinear processes of elections with two selective subjects. Georgian Electronic Scientific Journal: Computer Science and Telecommunications, 2015, № 2(46), pp. 61-78.

[20] Sulava L. O. Mathematical and computer modeling of nonlinear processes of elections. Works of the International conference "Information and Computer Technologies, Modelling, Management" devoted to the 85 anniversary since the birth of I. V. Prangishvili, Tbilisi, 2015, by p. 387-390 (russian).

[21] Chilachava T. I., Sulava L. O. Mathematical and computer simulation of processes of elections with two selective subjects and float factors of model. Problems of security management of difficult systems. Works XXIII of the International conference, Moscow, 2015, p. 356-359 (russian).

[22] Chilachava T. I., Sulava L. O. Mathematical and computer modeling of three-party elections. GESJ: Computer Sciences and Telecommunications, 2016, № 2 (48), pp. 59-72.

[23] Chilachava T. I. Nonlinear mathematical model of bilateral assimilation Georgian Electronic Scientific Journal: Computer Science and Telecommunications, 2014, № 1(41), pp. 61-67.

[24] Chilachava T. I., Chakaberia M. R. Mathematical modeling of nonlinear process of assimilation taking into account demographic factor. Georgian Electronic Scientific Journal: Computer Science and Telecommunications, 2014, № 4 (44), pg. $35-43$.

[25] Chilachava T. I., Chakaberia M. R. Mathematical modeling of nonlinear processes bilateral assimilation, Georgian Electronic Scientific Journal: Computer Science and Telecommunications, 2015, № 2(46), pg. 79-85.

[26] Chilachava T. I., Chakaberia M. R. Mathematical modeling of nonlinear processes of two-level assimilation, Georgian Electronic Scientific Journal: Computer Science and Telecommunications, 2016, № 3(49), pg. 34-48.

[27] Chilachava T., Gvinjilia Ts. Nonlinear mathematical model of dynamics of processes of cooperation interaction in innovative system. VII International Conference of the Georgian mathematical union, Book of Abstracts, Batumi, 2016, pp. 104-105.

[28] Chilachava T., Gvinjilia Ts. Nonlinear mathematical model of interaction of fundamental and applied researches, Problems of security management of difficult systems. Works XXIV of the International conference, Moscow, 2016, pp. 289-292 (russian). 